\title{
Handwriting Character Classification Using Freeman’s Olfactory KIII Model
}

\author{
Masanao Obayashi*, Shinnosuke Koga*, Ling-Bing Feng*, \\ Takashi Kuremoto*, and Kunikazu Kobayashi* \\ *Graduate School of Science and Engineering, Yamaguchi University \\ 2-16-2 Tokiwadai, Ube, Yamaguchi, 755-8611, Japan \\ \{m.obayas,n017vk, n007we, wu, koba\}@yamaguchi-u.ac.jp) \\ Home Page: http://www.nn.csse.yamaguchi-u.ac.jp/
}


Abstract- Recently, researches on smell sense that is one of the sensory organs of human have been actively done. The KIII model is one of the olfactory models that is thought out by Freeman referring to a physiological structure of mammal's olfactory system. In this paper, we propose a commonly used feature extraction method that applies Fourier transformation to time series behaviors of the internal state of M1 nodes in KIII, and also propose to use the dynamics of chaotic neuron instead of the Hodgkin-Huxley equation to reduce computation time. Our introduced structure of the chaotic neuron has the simple structure and that it makes possible the chaotic operation same as the Hodgkin-Huxley equation. Paying attention to the point that the human brain does a similar processing in any sense of information, the hand-written image recognition problem that uses the KIII model is adopted as the computation simulation. Through the computer simulation of the handwriting character classification, it is shown that the proposed method is useful in the point of both computation time and the recognition accuracy.

Key Words - Handwriting character classification, Freeman, KIII model, Fourier transformation, Chaotic neuron 


\section{INTRODUCTION}

Recently, according to development of non-invasive measurement techniques (CT, PET, fMRI, NIRS etc.), experimental research on higher-order brain functions has been advancing dramatically and the latest researches have been used in many fields. One of those high-order systems, the olfactory system, has also come to be actively done. As a model of the olfactory system, there is KIII model proposed by Freeman et al. [1] [3].

The KIII model has been modeled based on the physiological structure of the mammalian olfactory system, and there are many applications using pattern recognition ability of the olfactory system. For example, J. Fu et al. [2] combined KIII model and the electronic nose with chemical sensors, and applied it to discriminate six typical volatile organic compounds in Chinese wine. The KIII was also applied to Tea Classification problem (Yang et al. [4]), Face Recognition (Li et al. [6]). On the other hand, response of the olfactory system has a chaotic nature [5] [7], it has also attracted many researchers.

In this paper, we apply the KIII model to handwriting character classification. In the application of the KIII model mentioned above, they performed the pattern recognition by using standard deviation of time series response inside the KIII model corresponding to the inputted data which should be discriminated. In this paper, we propose to use Fourier transform as the feature extraction method.

On the other hand, in KIII model, output of the neuron is obtained by solving second order ordinary differential equations and as a result, that leads to the problem that enormous processing 
time is required. Therefore, in this study, we aim to reduce the processing time, maintaining the chaotic characteristic of each neuron, by adopting the simplified output formula.

\section{KIII MODEL}

KIII is a recurrent neural network model created by Freeman et al., based on biological olfactory structure [1]. The main parts of the olfactory neural system are composed of primary olfactory nerve (PON), olfactory bulb (OB), anterior nucleus (AON), and prepyriform cortex (PC). According to the anatomic architecture, KIII network is a multi-layer neural network model. Fig. 1 shows the structure of the KIII model, in which M and G represent mitral cells and granule cells in olfactory bulb respectively. $\mathrm{R}$ represents the olfactory receptor, which offers input to the KIII model. E, I, A, B represent excitatory and inhibitory cells in anterior nucleus and prepytiform cortex, respectively. The KIII model based on the olfactory neural system is a high dimensional chaotic network. In this model, the interaction of connected nodes leads to a high-dimensional chaotic attractor. After learning from different patterns, the system will form several low-dimensional local basins [3]. Therefore, the memory for different patterns might be regarded as the formation of the local basins, while the recognition process refers to the transition from one basin to another.

The parameters of the KIII model, such as connection weights between different nodes, were optimized to fulfill features observed in lots of electro-physiological experiments [3]. Every node is described as a second order differential equation as follows:

$$
\frac{1}{a b}\left[\ddot{x}_{i}(t)+(a+b) \dot{x}_{i}(t)+a b x_{i}(t)\right]=\sum_{j \neq i}^{N} w_{i j} Q\left(x_{j}(t), q_{j}\right)+k_{i} r_{i}(t),
$$




$$
\begin{aligned}
& Q(x(t), q)=\left\{\begin{array}{cc}
q\left(1-\exp \left(-\frac{\exp (x(t))-1}{q}\right),\right. & x(t)>-x_{0} \\
-1, & x(t) \leq-x_{0}
\end{array}\right. \\
& x_{0}=-\ln \left(1-q \ln \left(1+\frac{1}{q}\right)\right) .
\end{aligned}
$$

$x_{i}(t)$ : state variable of the $i$ th node, $w_{i j}$ : the connection weight from $\mathrm{j}$ to $i$ th node, $a, b, q$ : constants derived by the electro-physiological experiments on biological olfactory system. $k_{i}$ : coefficient as to the effect of the input, $r_{i}(t)$ : input ( external stimuli), $Q(\cdot)$ : a static sigmoid function derived from Hodgkin-Huxley equation and evaluated by the experiments, ${ }_{N}$ : the number of neurons.

\section{FEATURE EXTRACTION}

Usually, when doing pattern recognition using the KIII model, we should create a feature vector from the characteristics of the behavior of M1 node dynamics.

\section{Feature extraction by the standard deviation (Conventional method)}

The state of OB layer mitral level is used as the strength of activity. The learning process only adjusts connection weights among the mitral. A modified Hebbian learning rule and a habituation rule are employed to learning of the KIII model.

When feature data are input to the KIII model with $\mathrm{n}$ channels, the behavior of each node is represented as an output time series as shown in Fig. 2. To get the characteristics of the $k$ th channel's state, a value $S D(k)$ is extracted. The period with input patterns is divided into $\mathrm{S}$ segments (Fig. 2) and $S D(k)$ is the mean standard deviations of these segments. $S D$, composed 
of all the $S D(k)$ in the OB layer, depicts the activities of the all channels and $S D_{m}$ is mean feature of the whole OB layer.

$$
\begin{aligned}
& S D(k)=\frac{1}{s} \sum_{r=1}^{s} S D_{k r}, k=1,2, \ldots, n \\
& S D_{m}=\frac{1}{n} \sum_{k=1}^{n} S D(k), S D=[S D(1), S D(2), \cdots, S D(n)] .
\end{aligned}
$$

According to the modified Hebbian learning rule Eq. (6), the connection weights are adjusted.

$$
\begin{gathered}
\text { IF } S D(i)>(1+K) S D_{m} \quad \text { AND } \quad S D(j)>(1+K) S D_{m} \\
\text { THEN } \quad w_{i j}^{\prime}=h_{H e b}, \quad w_{j i}^{\prime}=h_{H e b} \\
\text { ELSE } \quad w_{i j}^{\prime}=h_{h a b} w_{i j}, \quad w_{j i}^{\prime}=h_{h a b} w_{j i},
\end{gathered}
$$

$w_{i j}, w_{i j}^{\prime}$ : connection weights between M1 nodes before change and after one, respectively, $K$ : bias, $h_{\text {неb }}$ : strengthening coefficient for Hebb learning, $h_{\text {hab }}$ : weakening coefficient for refinement learning. The learning for connecting weights continues while it is under changing.

\section{Fourier transform feature extraction method (Proposed method)}

Feature vectors are extracted as power spectrum and their frequency obtained by the discrete Fourier transform of time-series data of M1 node as shown in Fig. 3. It shows an example of the power frequency spectrum.

\section{SIMPLIFICATION OF THE OUTPUT NONLINEAR FUNCTION OF THE NODE}


The calculation of solutions of second order differential equations for determining the output of the neurons requires enormous processing time. This is a common problem in conventional method. Therefore, to reduce the computation time, we intend to simplify the second order differential equation. Furthermore, to produce chaotic response which is in the response of the above mentioned equations, we propose two simplified equations referring to dynamics Eq. (7) and Eq. (8), which are used in chaotic neural network (CNN) proposed by Aihara et al. [8].

\section{Dynamics of chaotic neural networks}

The dynamics of the $i$ th chaotic neuron of chaotic neural networks is as follows,

$$
\begin{aligned}
& y_{i}(t+1)=k y_{i}(t)+\sum_{j=1}^{N} \omega_{i j} x_{j}(t)-\alpha x_{i}(t)+r_{i}(t) \\
& x_{i}(t+1)=f\left(y_{i}(t+1)\right)
\end{aligned}
$$

Here, $y_{i}(t)$ : internal state of the $i$ th neuron at time $t, x_{i}(t)$ : output of the $i$ th neuron, $w_{i j}$ : connection weight from $j$ th neuron to $i$ th neuron, $k$ : refractory decay coefficient, $\alpha$ : constant parameter, $r_{i}(t)$ : input (external stimuli), $N$ : the number of neurons in the networks, $f:$ output function of a neuron.

We consider next two simplified chaotic neuron dynamics instead of Eq. (1) - (3) of KIII.

\section{$\underline{\text { Simplified equation I }}$}

$$
y_{i}(t+1)=k y_{i}(t)+\sum_{j \neq i}^{N} \omega_{i j} Q\left(x_{j}(t), q_{j}\right)-\alpha x_{i}(t)+r_{i}(t)
$$




$$
x_{i}(t+1)=\frac{1}{1+\exp \left(-\frac{y_{i}(t+1)}{\varepsilon}\right)} .
$$

$\underline{\text { Simplified equation II }}$

$$
y_{i}(t+1)=k y_{i}(t)+\sum_{j \neq i}^{N} \omega_{i j} x_{j}(t)-\alpha x_{i}(t)+r_{i}(t)
$$

\section{HANDWRITING CHARACTER CLASSIFICATION}

When performing the image pattern recognition using KIII model, there is a question of how to create input vectors of treating the image. In this research, two pre-treatment are carried to create the input vector.

\section{Pixel Average (PA) method}

By dividing the image to plural segments and taking the average of each pixel value of split images, we create input vectors which consist of them.

\section{Discrete Cosine Transform (DCT) method}

The DCT is one of ways to convert discrete signals into discrete frequency domain. It is mainly used for signal compression, in image processing, that is, the technique has been used in image compression such as MPEG and JPEG. Compared to the discrete Fourier transform, this is characterized by the spectrum tend to be concentrated in specific frequencies. In the DCT, the 
DCT coefficients, that is, features in this research, are calculated by DCT basis functions and they are divided to the directional component (DC) and alternating component (AC). If you make the image DCT transform, the low frequency components (coefficients) are greater, conversely, high-frequency components become so smaller. The formula of DCT is shown in Eq. (12).

$$
\begin{aligned}
& F_{k, l}=\sum_{j=0}^{N-1} \sum_{i=0}^{N-1} f_{i j} \phi_{k}[i] \phi_{l}[j] \\
& f_{k, l}=\sum_{l=0}^{N-1} \sum_{k=0}^{N-1} F_{k l} \phi_{k}[i] \phi_{l}[j] \\
& \phi_{k}[i]= \begin{cases}\frac{1}{\sqrt{N}} & k=0 \\
\sqrt{\frac{2}{N}} \cos \frac{(2 i+1) k \pi}{2 N} & k=1,2, \cdots, N-1\end{cases}
\end{aligned}
$$

We show the proposed system in Fig. 7.

\section{COMPUTER SIMULATION}

We confirm the usefulness of the proposed method under the conditions as follows,

- Data format : bmp,

- Data size : $512 \times 512$,

- Subject : 15 (Male),

- The number of channels : 64

- The number of Japanese characters (refer to Fig.8) :

$$
\begin{aligned}
& 200(=10 \text { males } \times 4 \times 5 \text { characters for training }), \\
& 100(=5 \text { males } \times 4 \times 5 \text { characters for testing })
\end{aligned}
$$

$\underline{\text { Simulation } 1}$ 
We first verify usefulness of the Fourier transform comparing with the standard deviation. Simulation results are shown in Table 1. The method using PA \& FT is better than the conventional one can be confirmed by the results.

\section{$\underline{\text { Simulation } 2}$}

We next verify usefulness of two cases of simplified output functions of neurons comparing with the conventional second-order differential equation. Simulation results are shown in Tables 2 , 3 and 4.

\section{DISCUSSION AND CONCLUSION}

Recognition result of the feature vector extraction method by the Fourier Transform showed higher recognition rate than the conventional method, i.e., by the Standard Deviation. In addition, processing times of the simplified two output functions of neurons introduced to make the enormous processing time of KIII model reduce are shortened by about $1 / 150$ of the conventional for the simplified formula I, about 1 / 5 of the conventional for Formula II. However, the recognition rate of both simplified output functions came to be reduced.

Performance improvement of the proposed method making appropriate trade-off between improving the recognition rate and increasing the processing time, as increasing the number of dimensions of feature vectors, is remained as the future work.

\section{REFERENCES}

[1] Hung-Jen Chang, Walter J. Freeman, Brian C. Burke, Optimization of olfactory model in 
software to give 1/f power spectra reveals numerical instabilities in solutions governed by aperiodic (chaotic) attractors, Neural Networks 11, pp449-466 (1998)

[2] Jun Fu, Guang Li, Yuqi Qin, Walter J. Freeman, A pattern recognition method for electronic noses based on an olfactory neural network, Sensors and Actuators B 125, pp485-497 (2007)

[3] Chang. H.J., Freeman W.J. Biologically Modeled Noise Stabilizing Neurodynamics for Pattern Recognition. Int J of Bifurcation and Chaos, 8(2) pp.321-345 (1998)

[4] Xinling Yang, Jun Fu, Zhengguo Lou, Liyu Wang, Guang I, Walter J. Freeman, Tea Classification Based on Artifcial Olfaction Using Bionic Olfactory Neural Network, NCS,pp.343-348 (2006)

[5] W.J. Freeman, Simulation of Chaotic EEG Pattern with a Dynamic Model of the Olfactory System, Biol. Cybern. 56,pp.139-150 (1987)

[6] Guang Li, Jin Zhang, You Wang, Walter J. Freeman, Face Recognition Using a Neural Network Simulating Olfactory Systems, LNCS 3972, pp.93-97 (2006)

[7] C.K. Skarda, W.J.Freeman,How brains make chaos in order to make sense of the world, Behavioral And Brain Science, Vol. 10, pp.161-195 (1987)

[8]K. Aihara, T.takabe and M.Tomita, Chaotic Neural Networks, Physics Letters A, 144, pp.133-340(1990) 


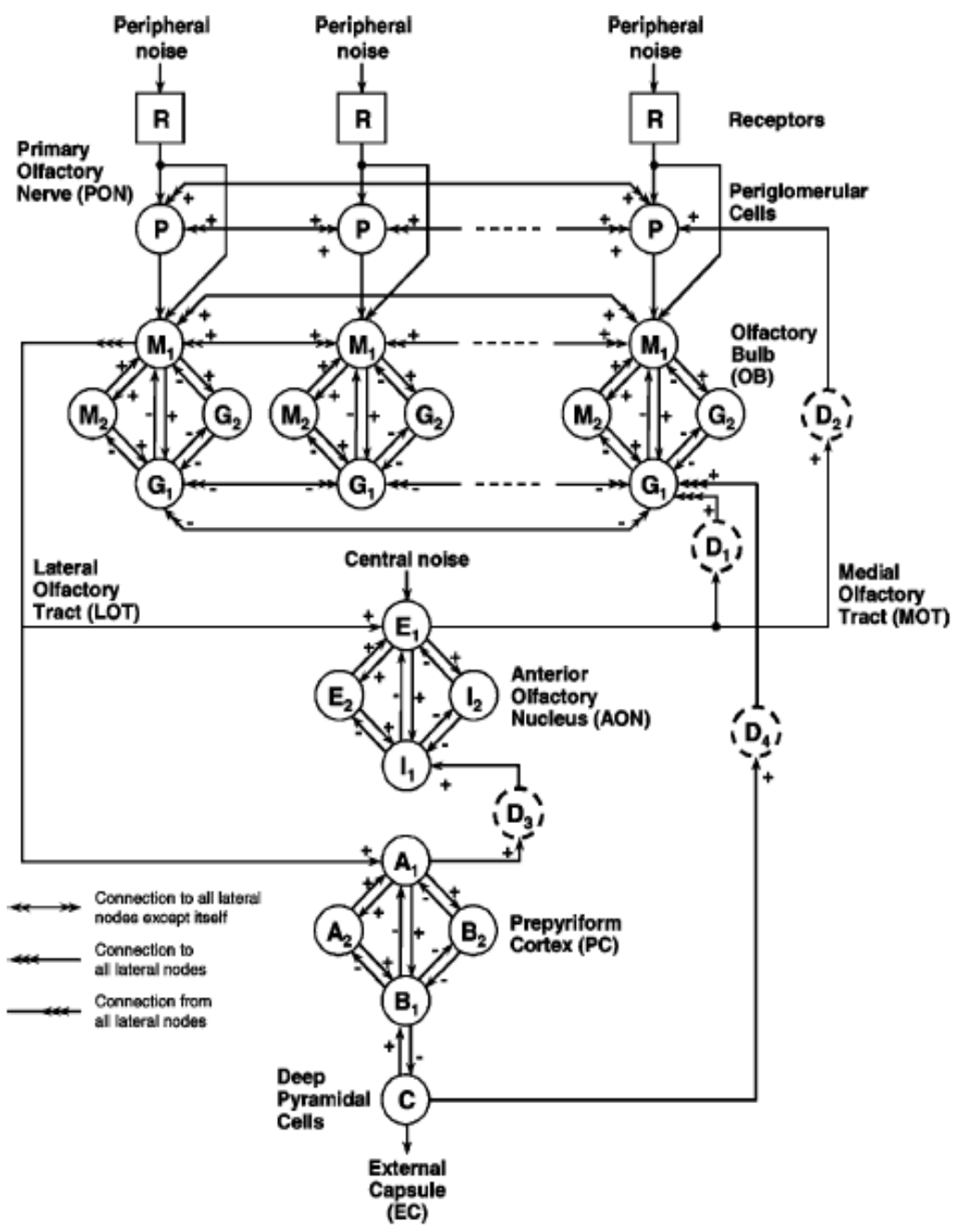

Fig. 1 Structure of the KIII Model (from [5][6]) 


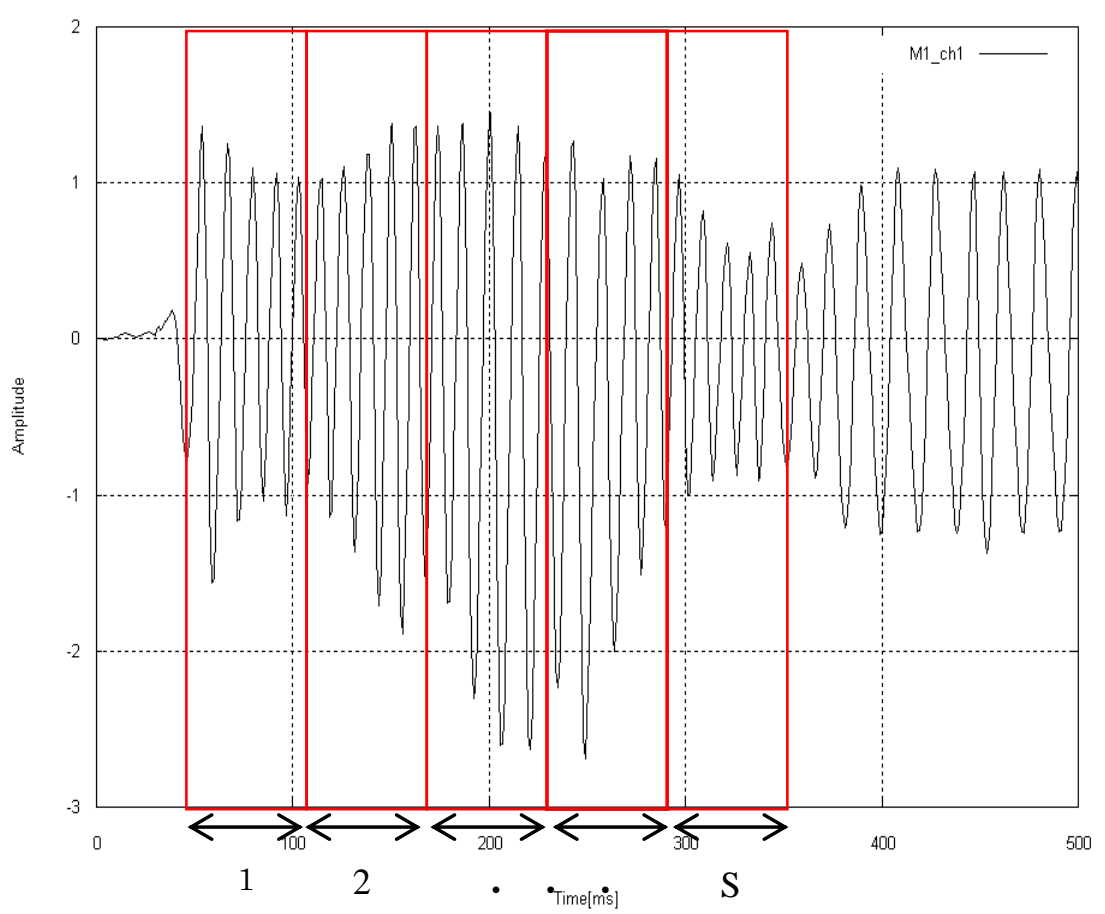

Fig. 2 Example of segmentation in the time-series behavior of the internal state of a M1 node 


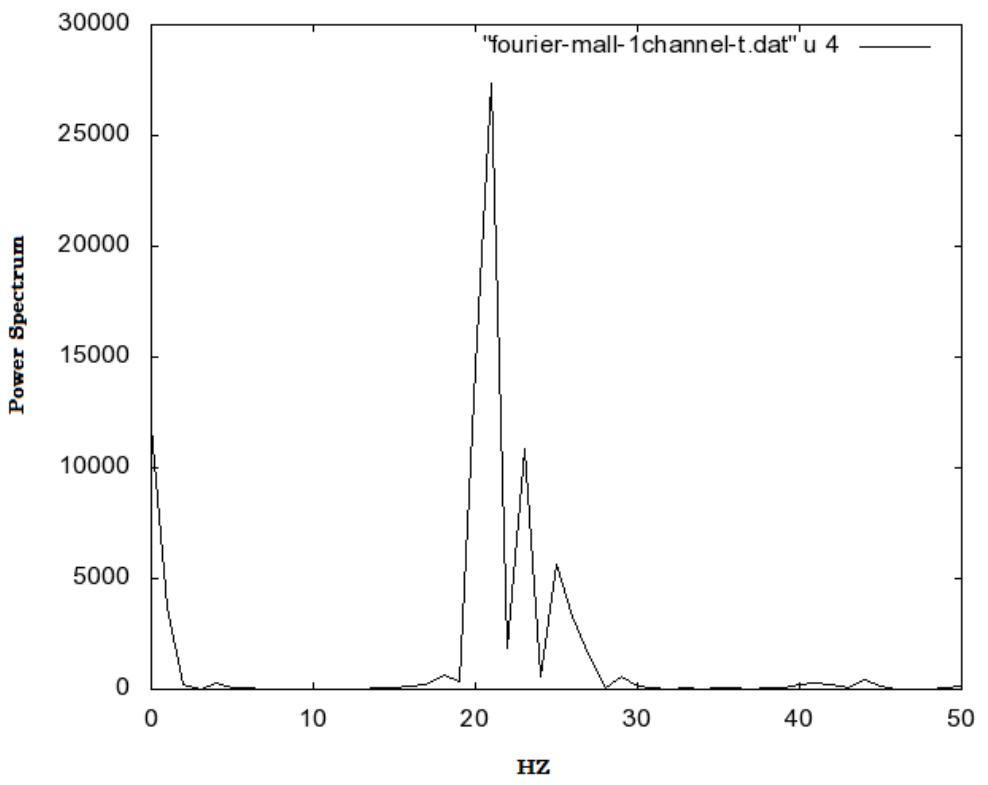

Fig.3 An example of power spectrum 


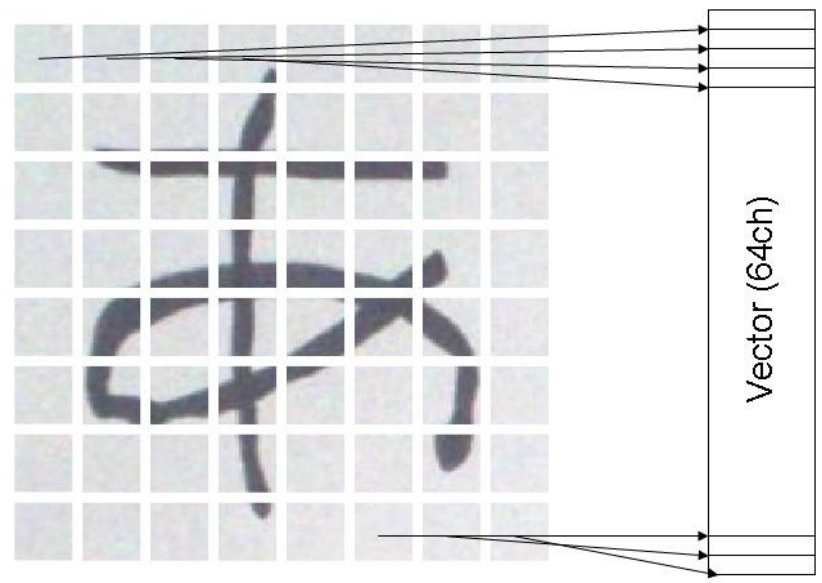

Fig. 4 Example of image segmentation 


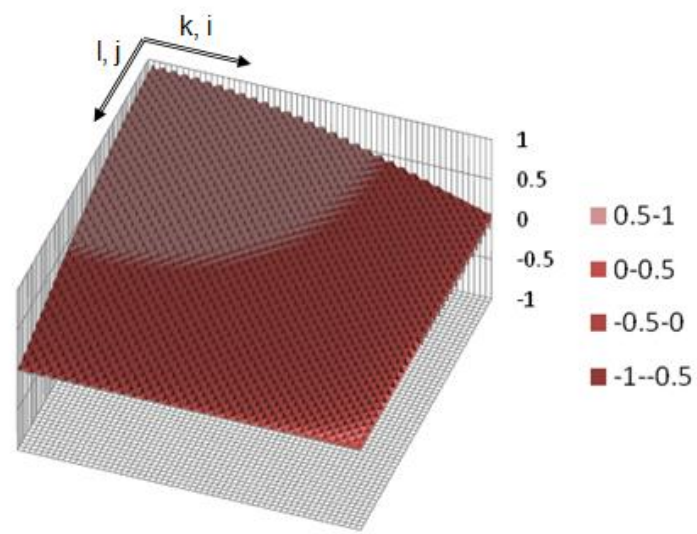

Fig.5 Values of DCT basis function $(64 \times 64) \phi_{k}[i] \phi_{l}[i]$ 


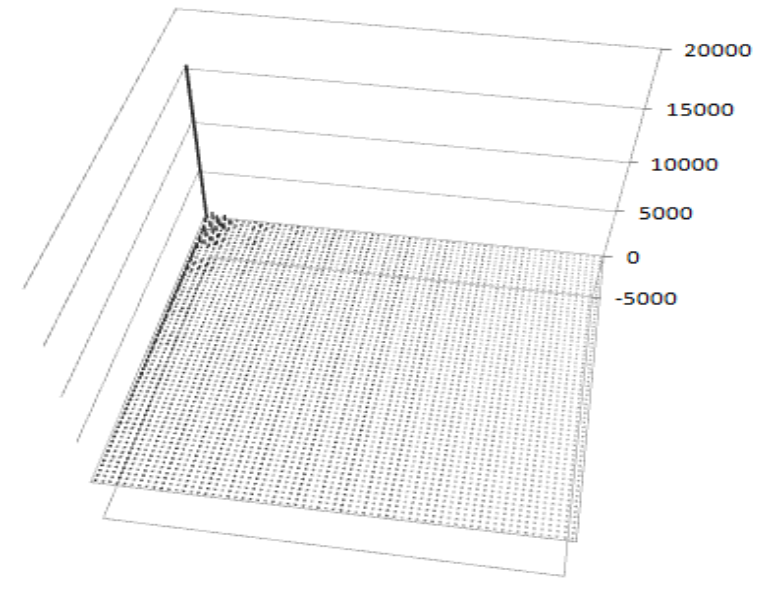

Fig.6 Example of DCT coefficients (64×64) 
Input vector with M ch (pixel averages / DCT coefficients )

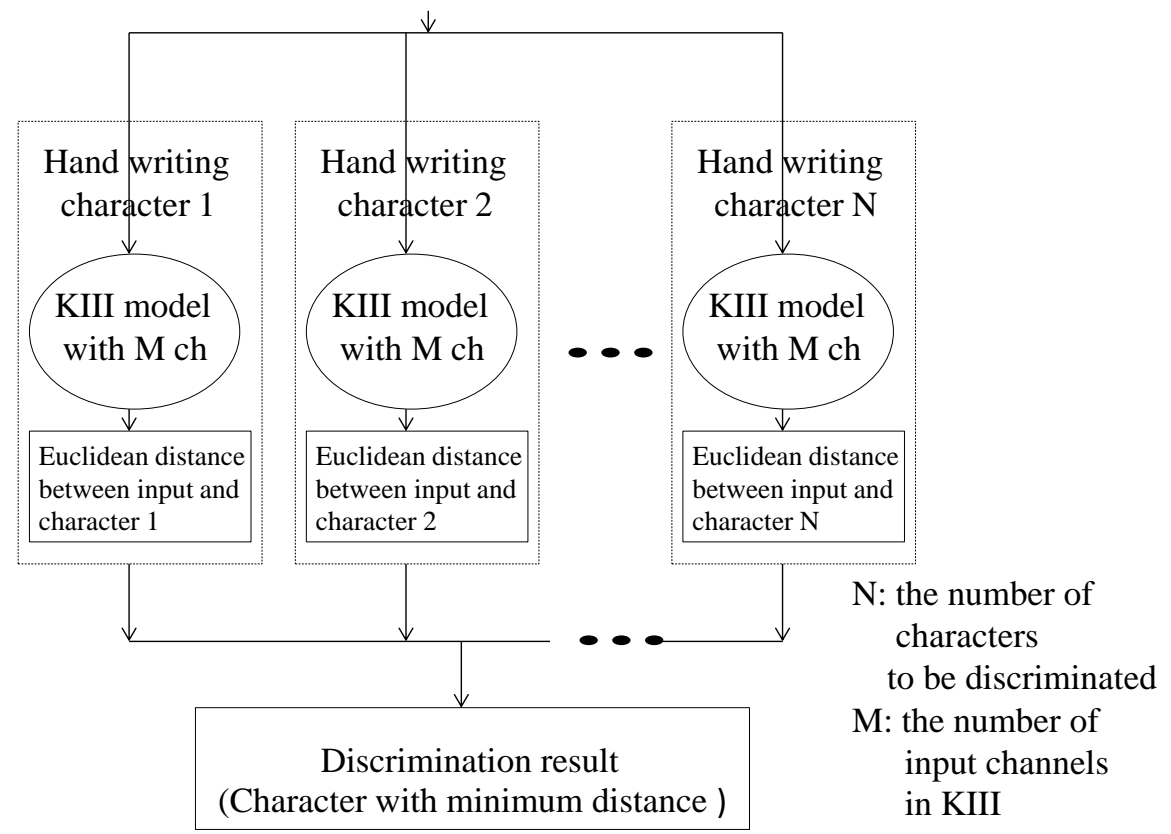

Fig. 7 Hand writing recognition system using KIII model 


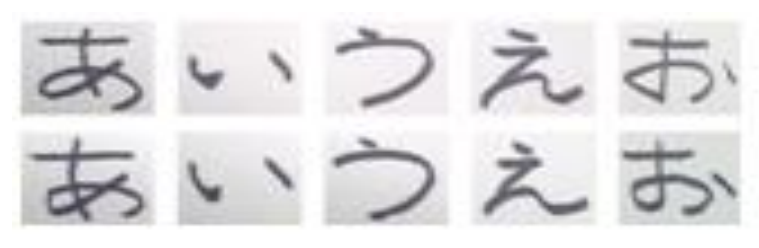

Fig. 8 Examples of hand writing images used in simulation 
Table 1 Success rates of 2 feature extraction methods (\%)

\begin{tabular}{|c|c|c|}
\hline Model & Standard & Fourier \\
\hline Input feature & Deviation & Transform \\
\hline extraction method & & \\
\hline PA method & 89.0 & 94.0 \\
\hline DCT method & 84.0 & 91.0 \\
\hline
\end{tabular}


Table 2 Success rates of the simplified KIII model I (\%)

\begin{tabular}{|c|c|c|c|c|}
\hline \multirow{2}{*}{$\begin{array}{l}\text { Input feature } \\
\text { extraction } \\
\text { method }\end{array}$} & \multicolumn{2}{|c|}{$\alpha=1.0$ in } & \multicolumn{2}{c|}{$\alpha=0.35$ in Eqs. } \\
\cline { 2 - 5 } & Eqs.(6), (7) & \multicolumn{2}{|c|}{$(6),(7)$} \\
\hline PA method & 51 & 62 & 47 & 79 \\
\hline DCT method & 27 & 58 & 37 & 62 \\
\hline
\end{tabular}


Table 3 Success rates of the simplified KIII model II (\%)

\begin{tabular}{|c|c|c|c|c|}
\hline \multirow{2}{*}{$\begin{array}{l}\text { Model } \\
\text { Input feature } \\
\text { extraction } \\
\text { method }\end{array}$} & \multicolumn{2}{|c|}{$\alpha=1.0$ in } & \multicolumn{2}{c|}{$\alpha=0.35$ in } \\
\cline { 2 - 5 } & Eqs.(6), (7) & \multicolumn{2}{|c|}{ Eqs.(6), (7) } \\
\hline PA method & 47 & 68 & 60 & 61 \\
\hline DCT method & 40 & 59 & 34 & 69 \\
\hline
\end{tabular}


Table 4 Computation times among 3 methods

\begin{tabular}{|c|r|}
\hline Model & time(sec) \\
\hline KIII model & 3034 \\
\hline Simplified KIII model I & 640 \\
\hline Simplified KIII model II & 24 \\
\hline
\end{tabular}

